\title{
Passive Indoor Positioning System (PIPS) Using Near Field Communication (NFC) Technology
}

\author{
Stephen Tang ${ }^{1}$, BeeLing Tok and Martin Hanneghan ${ }^{2}$ \\ Department of Computer Science, \\ Faculty of Engineering and Technology, \\ Liverpool John Moores University, \\ Byrom Street, Liverpool, L3 3AF, UNITED KINGDOM. \\ O.T.Tang@ljmu.ac.uk ${ }^{1}$ and M.B.Hanneghan@ljmu.ac.uk ${ }^{2}$
}

\begin{abstract}
Travel can be an enjoyable experience but it can also be stressful when one is unable to get to the destination in timely manner. Satellite navigation systems (satnav) such as the ubiquitous Global Positioning System (GPS) provide an aid to locating unfamiliar places without hassle. However, the effectiveness of satnav stops at the doorstep of the building due to its requirement for line of sight with orbiting satellites. Within a large complex building, navigation typically relies on building signage, information from kiosks and getting assistance from information desks. The advancement of mobile devices and wireless technology offer an interesting proposition for the development of indoor positioning systems. In this paper, we propose a passive indoor positioning system to provide navigational aid and discuss findings from our pilot experiment using NFC technology.
\end{abstract}

Keywords-Passive Indoor Positioning System; Positioning System; Mobile Application; Mobile Technologies; NFC Technologies;

\section{INTRODUCTION}

"James works as a sales person for a company. Every day, he meets with new clients trying to secure new sales. He works hard to meet his sales target and he is very good at his job. One day, he was asked at very short notice to meet an important client at certain time in a building and a location with which he was not familiar. Problems in locating the venue meant that James missed the important meeting and subsequently missed his sales target for that quarter. With this in mind, he has been constantly on the lookout for a solution similar to GPS (Global Positioning System) for use inside a building. Until finally, he was introduced to newly designed building that has an indoor positioning system. Ever since that day, he hopes that all public buildings are installed with indoor positioning system so that he does not have to get lost again."

Getting lost in an unfamiliar place is a typical experience that occurs to many of us. In the past, travellers had a strong sense of direction and would rely on people that are familiar with the place to provide useful navigational guide to help us our destination. In the present day, with the advancement of technology, we are able to get a lot of information about a place either via the Internet or using navigation aids such as GPS which are built into most smart devices. People can now get to places easily without much preparation or plan for the journey ahead (provided that the technology is working). In the market surveys published by Ofcom [1-2] on the activities conducted on smartphones by teenagers and adults in the UK in 2011, the use of online maps or GPS is listed as one of the regular functions accessed on the smartphones. In total, 5\% of teenagers used the map or GPS function regularly and another $15 \%$ have used the function. The percentage is higher in adult users where $18 \%$ use the map or GPS function regularly and a further $26 \%$ have used it before. The increased need for navigational aid in adults could be explained by the increased opportunity to travel both for work and leisure.

GPS technology is able to provide location information in open space only and requires line-of-sight to operate. GPS signals are weak in indoor and within harsh environments, which could reduce the accuracy of GPS and make satellites undetectable. The need for navigational guidance inside a large and unfamiliar building is still necessary even though the scale of the area is considerably smaller than the outside environment. In large organisations such as public administration buildings, Universities or hospitals, the networks of walkways could have similar complexity to urban road networks. A lack of, or poor signage and foreign language instructions could also add to the challenge.

In the recent years, the Internet of Things (IoT) has gained significant attention from the industry and research communities. IoT refers to a network of physical objects which has embedded components such as electronics, software, sensors and connectivity to communicate data either from the internal states of the object or the external environment to another computing device [3]. IoT has convergence with wireless technologies, micro-electro-mechanical devices and the Internet. When IoT objects sense and communicate, it changes how and where decisions are made and, who makes them [3].

In this paper, we intend to address the issues pertaining to indoor navigation by taking advantage of IoT technologies and integrating this with existing smart devices by creating a passive indoor positioning system (PIPS). In Sections II and III, we review the conventional approaches used to aid people 
to navigate or plan their navigation inside a building and survey the use of local-range signals technologies for indoor positioning. In Section IV, we outline the system architecture for PIPS and discuss the various wireless identification standards such as Near Field Communication (NFC) and Bluetooth Low Energy (BLE) which can be used as digital tags within the environment in which PIPS operates. The scope and features of PIPS for the Department of Computer Science at Liverpool John Moores University is presented in Section V. The details and findings from the experiment that evaluates PIPS are presented in Section VI. Finally, Section VII concludes and presents the future work related to this project.

\section{REVIEW OF CONVENTIONAL APPROACHES TO SUPPORT INTERIOR NAVIGATION}

Navigation involves ascertaining the position of a location, planning a route to take to arrive at the destined location and monitoring the planned route. In the context of interior navigation, typical solutions made available to visitors include floor plan, local signage, interactive kiosks, customer service staff and Location Based Services (LBS).

Wayfinding and cognitive maps are natural human abilities to navigate within our environment. Wayfinding refers to the natural process where an individual actively locates their destination, plans the route and then pursues the route. This wayfinding behaviour allows us to develop cognitive maps that represent spatial information of the environment such as points of interest and routes. There is a strong correlation between the level of difficulty for wayfinding and the complexity of a spatial (and virtual) environment [4-5]. The architecture and design of a building plays an important role in influencing wayfinding behaviour [5-6]. A well-designed building layout can make wayfinding an easy task which can reduce overall locomotion time and improve visitors' experience to get to a destination inside a building or even exiting the building in case of emergency. While cognitive mapping is developed over time by us through our wayfinding activity in a spatial environment, a floor plan of a building can be made available as a graphical representation to provide an instant cognitive map of the spatial environment. This is a simple and economical solution to support indoor navigation. However, the complexity of a floor plan has influence over the clarity with which individuals develop their own cognitive map and this will have an impact on the effectiveness of wayfinding [7].

Local signage is another non-technological solution that supports indoor navigation. Signage is designed as a label for a location or to provide a directional guide to a point of interest. It can be in the form of a graphical symbol, text or both. The symbols for most inside building signage are usually derived from the ISO 7001 standard for public information symbols [8] or could be created by adhering to the ISO22727 standard for creation and design of public information symbols requirements [9]. These are typically placed in specific points inside a building as a navigational aid to visitors. Local signage can be a very effective navigational guide to visitors if the symbols and text used are representative and communicative, and the signage itself is well placed. However, there is still a possibility of misplaced signage or confusing translated captions used on the signage.

Information centres or reception counters can also be regarded as another form of non-technology related solution for indoor navigation. Customer service staff or receptionists are able to help out with any enquiries posed by visitors relating to details about product, services or even providing navigational instructions to visitors. However, there could still be factors contributing to the unpleasant experience of having difficulty finding a location inside a building where staff at the information centres may be inexperienced.

In modern buildings, technological solutions such as interactive kiosks provide a means for visitors to search the directory of a building and some can provide users with directional information from the interactive kiosk to the required location within the building [10]. The information is typically contained within a stand-alone kiosk at a fixed location. It displays the current location and people are able to search for their destination. If the interactive kiosks are networked, information is transmitted electronically to other sites to update the database [11]. Interactive kiosks are used to minimize the needs of receptionists or customer service personnel. Unlike traditional floor plans which present all the necessary information on a single visual representation, interactive kiosks only present a subset of information. This would require the user to make repeated attempts to refine their query in order to obtain the necessary direction to a location within the building. This therefore suffers from the same issues as static floor plan solutions discussed earlier.

LBS are mobile service information points that extend the information to end users via Internet and wireless communication. LBS track and use location data to push relevant information to users' mobile devices either through Short Messaging System (SMS) or via installed apps [12-13]. Prior to GPS chips being embedded into mobile devices, the approximate position of a mobile phone is computed from trilateration with signal analysis from known base stations. Relevant messages can be received via Wireless Application Protocol (WAP) or SMS and could then be pushed out to known mobile devices in the area [14]. This positioning approach which constantly scans for a device's position can be costly and less accurate due to the size of area covered making it less viable as a technique for indoor navigation assistance. LBS is used mostly as a medium to provide location specific information or marketing.

\section{REVIEW OF EXISTING NEAR LBS (NLBS) TECHNOLOGIES FOR INDOOR POSITIONING TO SUPPORT INTERIOR NAVIGATION}

As mobile devices become more advanced, more wireless technologies are embedded within small devices. This provides an opportunity to explore how these wireless technologies can be used for indoor navigation. The newer approach known as Near LBS (NLBS) uses local-range signals such as Wi-Fi, BLE and NFC technologies to determine the position of a mobile device and can be used to develop indoor positioning systems.

A Wi-Fi-based positioning system uses Wi-Fi signals from network access points to create a radio map which is used with a mobile app that scans signal strengths from multiple access point to determine the position of a mobile device in the physical space [15]. This approach is only cost effective for buildings that have multiple $\mathrm{Wi}-\mathrm{Fi}$ access points installed to provide signal coverage for the whole of the building.

Another application of indoor positioning which uses similar setup to the Wi-Fi-based positioning system involves the use of Radio Frequency Identification (RFID) technology. $m$ Tag is an example of RFID-based indoor positioning system which uses stationary RFID readers placed within a building with a passive RFID tag attached to a mobile device [16]. It replaces Wi-Fi access points with RFID readers linked up with a centralized system to provide positional information via web services. This enables users to obtain near real time positioning 
data via a mobile web app. The downside of this solution is the cost involved to set up the infrastructure.

In the recent years, BLE has gained popularity in personal and wearable computing. BLE, also known as Bluetooth Smart or Bluetooth 4.0, is a wireless personal area network technology that uses low power consumption while still operating at a similar communication range to earlier revisions. BLE allows the enhancement of dumb devices with Bluetooth wireless technology that are powered by coin-cell batteries. The features of BLE are ultra-low peak, average and idle mode power consumption, low cost, ability to run for years on standard coin-cell batteries, multi-vendor interoperability and enhanced range [17]. BLE devices such as Estimote Beacon [18], StickNFind [19] and Tile [20] are used for contextual awareness [17], tracking personal items [18-21] and could even be used for indoor positioning purposes [18-19]. BLE-based positioning systems use the signal strengths from multiple beacons placed in physical spaces to analyse and compute the approximate position of a mobile device. This is a relatively low cost compared to mTag. However, the signal strengths vary depending on environmental factors such as obstacles and therefore can only provide an approximation of distance [22].

Although both the Wi-Fi-based positioning system and BLE-based positioning system are useful and relatively easy to deploy systems, the cost involved is still considerably high. In addition to that, both systems are highly reliant on radio map and triangulation computations based on radio signal strength which is always dependent on the environment in which the indoor positioning system is situated and the type of receiver(s) on which it operates [23]. One of the possible approaches to improve of indoor position tracking is to ensure the individual is checking in at a stationary point. In the next section, we describe the architecture for a passive indoor positioning system which is based on stationary checkpoints using markers.

\section{SySTEM ARCHITECTURE FOR PASSIVE INDOOR POSITIOING SYSTEM (PIPS)}

Our proposed PIPS is a simple and low cost system. A building is simply tagged with location markers and users use a mobile app to obtain navigational information of the building. A user of PIPS can either search for a specific location and direction using the app, or make use of the location marker as a starting point or as checkpoints en route to update the path shown on the mobile app.

The proposed PIPS consist of 3 key components; (1) location markers, (2) standalone mobile app and (3) mobile device (see Fig. 1). Users can use the PIPS mobile app installed on the mobile device to search for a navigational path from location A to location B. They can also make use of the location markers that are placed physically in public areas of a building as a starting location to the desired location or checkpoints en route to update their current position within their current navigational path.

Location markers are unique location identifiers which take the form of visual marker or radio frequency-based marker. The types of marker supported in the proposed system architecture are those that can be read using a camera or onboard connectivity technology such as NFC and Bluetooth in present day mobile devices. The use of location markers eliminates the need for typing in current location and therefore improves the effectiveness of this system. Examples of visual markers include QR Codes [24] and barcodes. Visual markers are relatively cheap to produce, can easily be integrated into wide range of print materials and can be scanned using the camera on mobile devices. Visual markers are suitable for close proximity and well illuminated environments. Radio frequency based marker such as NFC and BLE are alternatives to visual markers. These do not require line-of sight operation and can be operated at set range depending on type of radio frequency technology. RFID and NFC operate on the same technology and only vary in the read proximity. NFC tags can operate within less than $10 \mathrm{~cm}$ proximity with the NFC reader in order to register a successful read [25]. BLE is an alternative can be read using Bluetooth receiver at a distance up to 100 meters [26].

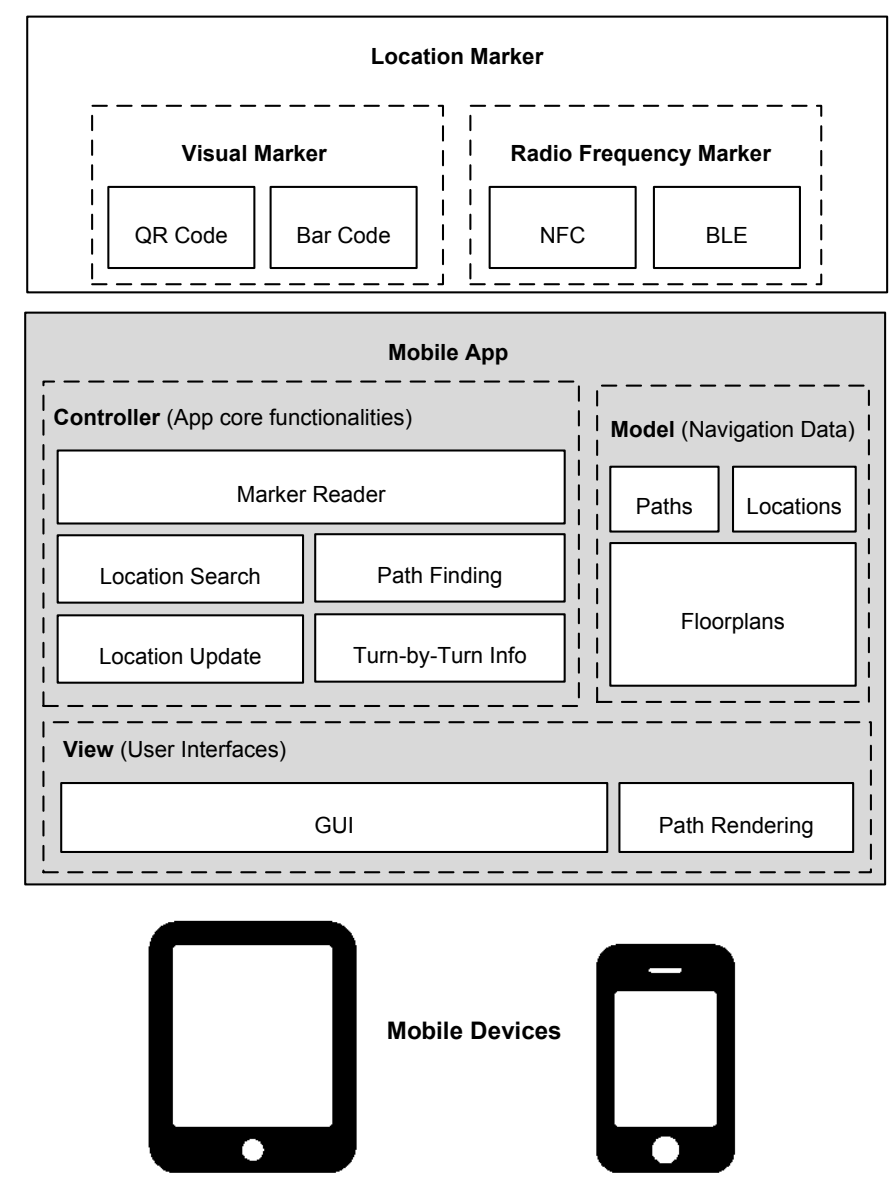

Fig. 1. System Architecture for PIPS.

The standalone mobile app for PIPS is designed using the Model-View-Controller (MVC) pattern. The Model component in PIPS consists of the crucial navigational data such as paths, locations and floorplan(s) for a building. The controller component includes the core functionalities of the mobile app that allows user to search for a location within a building, find the path to a destination, use the mobile device to read physical markers placed within the building to pinpoint the current location and use app to update position within the suggested route. The implementation of marker reader functionality is dependent on the type of marker used for the PIPS setup. Visual marker reading uses the camera with specific algorithms to interpret $\mathrm{QR}$ codes and barcodes, whereas radio frequency based markers make use of on-board connectivity protocols such as NFC or Bluetooth. The user interface to access these functionalities is presented via the View component using appropriate GUI and 2D draw methods for graphical path rendering.

In the next section, we describe our prototype implementation of PIPS for the Department of Computer Science at Liverpool John Moores University (LJMU). 


\section{PIPS FOR DEPARTMENT OF COMPUTER SCIENCE AT LJMU}

The Department of Computer Science (CompSci) at LJMU occupies two floors of a large multi-use building in the city centre. Floor directories can be seen at corridor level to provide visitors information about rooms and their occupants. The floor layouts are a combination of academic and research staff rooms and computing laboratories. All rooms are labelled with an identification plate consisting of room number and either occupant name or lab name.

Despite the simple layout of both floors, external visitors and new students unfamiliar with the site find it challenging to find particular rooms and/or staff easily. Many would still resort to wayfinding in an attempt to develop a cognitive map of the building but this would require time and exploration of which some visitors do not have the luxury of doing so. This is an ideal case to evaluate if PIPS could get visitors to their point of interest fast and easy. This is detailed in Section VI.

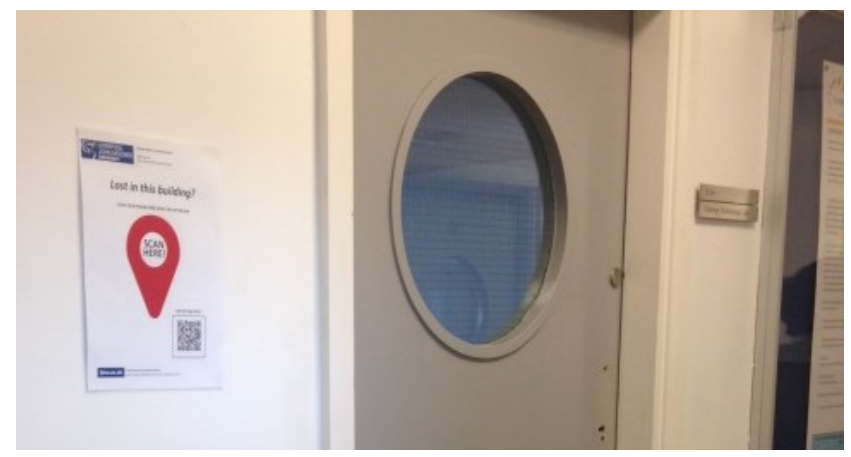

Fig. 2. NFC Location marker placed outside a room.

The PIPS implemented at LJMU uses NFC markers that are read using a mobile app developed on the Android platform. The location marker is simply a poster with NFC sticker. The NFC sticker used in the project is the NTAG216 NFC sticker with 888 byte capacity and operating distance of less than $2 \mathrm{~cm}$ manufactured by NXP Semiconductors ${ }^{1}$. The NFC location markers store a unique identifier as the node ID used to represent a specific point in the map (see fig. 4). These values were written using the NXP TagWriter ${ }^{2}$ app available from the Google Play Store. It is a cost conscious decision to use NFC technology as the location marker. The NTAG216N NFC sticker only costs from $£ 0.52$ (per every hundred). Although there are only a small proportion of NFC-enabled smartphones in the market at the moment, the installed base of NFC-enabled smartphones is growing and is estimated to reach 2.1 billion units by 2017 [27].

In order to provide a directional guide to user, the mobile app will need to know both the start point and the destination in order to seek the path. Users can provide the start point by manually selecting the nearest room number from the list, or read the NFC location marker placed outside of the room on one of the floors (see fig. 2). The destination can be entered either by selecting the room number from the list or searching for a specific person from the list (see fig. 3).

In order to compute a path from a given start point to a desired destination, a graph is used to represent paths and locations. Each room in a floor is represented as a node with a unique identifier in the graph and additional information such as room label and coordinate on the $2 \mathrm{D}$ map is also added.

\footnotetext{
http://www.nxp.com/products/identification_and_security /smart_label_and_tag_ics/ntag/series/NTAG213_215_216.html

${ }^{2} \mathrm{https} / /$ play.google.com/store/apps/details?id=

com.nxp.nfc.tagwriter\&hl=en GB
}

Additional nodes for every room were also added to represent outside of every room and some in the middle of the corridor. This is to facilitate drawing of an angled path as shown in fig. $2 \mathrm{f}$

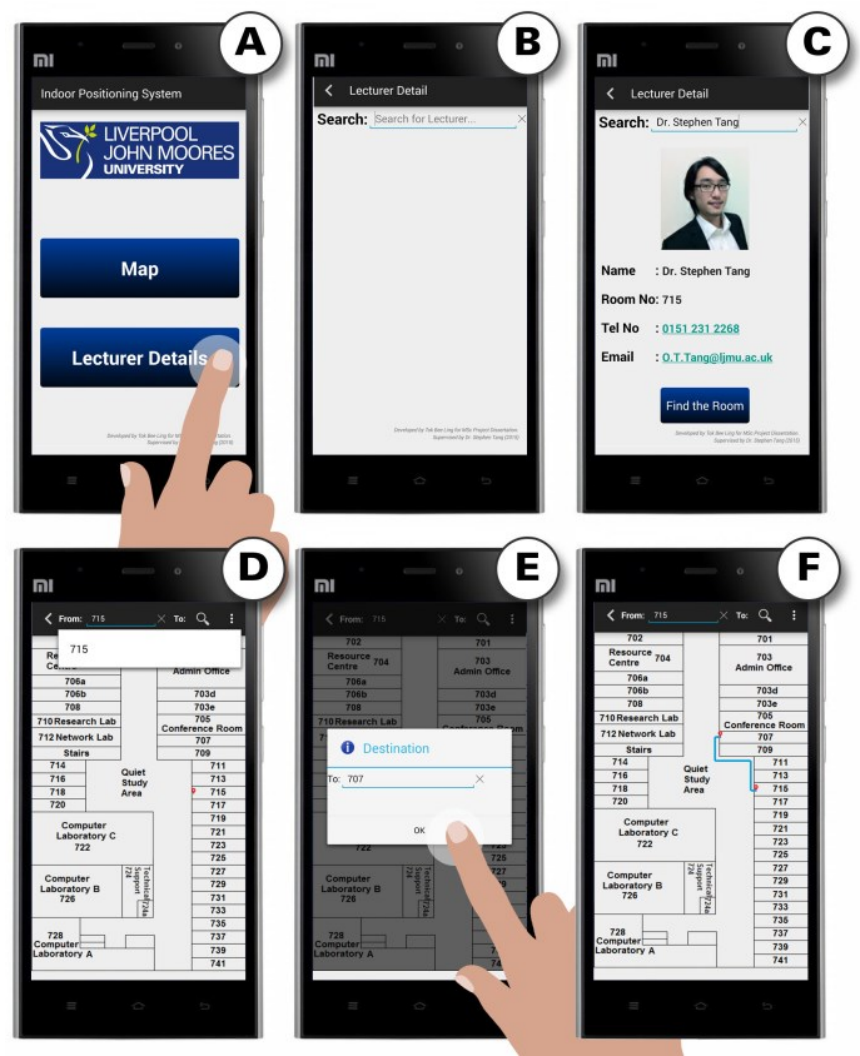

Fig. 3. Users can search for a person from the list and use it as a starting point to get direcitonal guidance to another room.

Nodes are then connected by defining the lanes to construct the graph as shown in fig.4. Each lane has a distance value which is used to find the shortest path. Each unit of distance represents approximately $30 \mathrm{~cm}(1 \mathrm{ft})$ in spatial distance. For every lane defined $(\mathrm{A} \rightarrow \mathrm{B})$, the reversed nodes lane $(\mathrm{B} \rightarrow \mathrm{A})$ is defined to ensure a path can be found if point $B$ is used as a starting point to go to point $\mathrm{A}$.

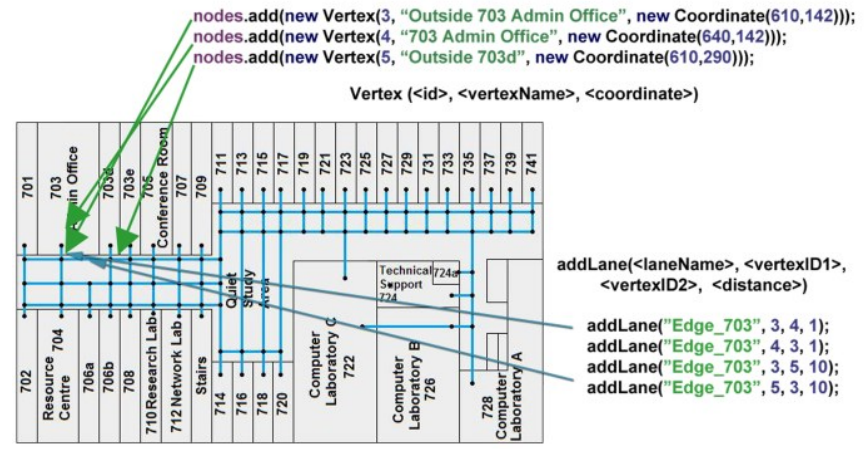

Fig. 4. Representation of nodes in the graph.

In order to search for a path in the graph constructed, given a start point and destination, we have chosen to use Dijkstra's algorithm. Dijkstra's algorithm finds the shortest path from source vertex to all other vertices in the graph. It then examines the closest to the source vertex and adds the vertex to the set of vertices to be examined. It expands outwards from the starting point until it reaches the goal. Once a path is found, coordinates of the nodes are used as vertices to draw edges which form a path [28]. 


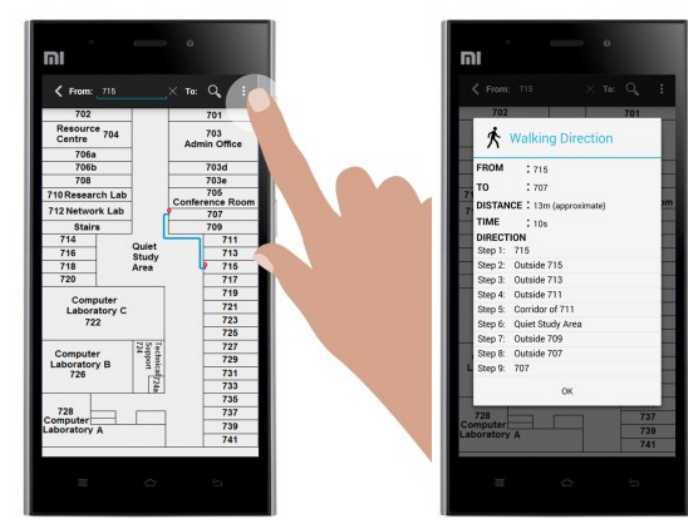

Fig. 5. Representation of nodes in the graph.

Each of the nodes found in the path are used as building mark to provide directional instruction to user as shown in fig. 4. The estimated time taken to walk from start point to destination is calculated based on the lower limit of human average speed of 3 miles (4.828032 kilometres) per hour considering most individual would walk slower inside a building [29]. This is computed using the following formula:

$$
\text { Duration in seconds }=\frac{\text { Distance in meter }}{(4828.032 \div 360)}
$$

\section{EVALUATION OF PIPS}

A pilot experiment was conducted to evaluate if the PIPS system could provide directional aid to users unfamiliar with the site. In this experiment, questionnaires were used to gather data pertaining to indoor wayfinding with and without PIPS and observations on the task were given.

\section{A. Experiment}

The study follows a controlled experiment design. A total of 16 participants were involved during the day of the experiment. Participants were divided into four experiment groups; (A) CompSci student with no PIPS assistance; (B) Non-CompSci student with no PIPS assistance; (C) CompSci student with PIPS assistance; and (D) Non-CompSci student with PIPS assistance.

Participants were given a short brief once they agreed to voluntarily take part in the experiment. They were given a preexperiment questionnaire to complete to gather background information regarding gender, age group, technology literacy, use of technology when planning navigation and experience navigating in a building. Participants were then given a room number or a lecturer at the Department which they needed to locate. Depending on the experiment group the participant had been assigned to by the observer, they may be given PIPS assistance. Participants with PIPS assistance are given a test mobile device (Xiaomi Mi3 Android smartphone) preinstalled with the PIPS mobile app. Participants were then left to complete the given task uninterrupted and were observed by the experimenter from a short distance.

At the end of the task, participants that used PIPS were asked to complete the post-experiment questionnaire which gathered data on user demographic and feedback on the usability and functionality of PIPS. Participants without PIPS assistance completed a different post-experiment questionnaire to gather insights into their past experience of being lost in a building and the approaches they use for wayfinding in an unfamiliar building.

\section{B. Findings and Analysis}

The pilot experiment was carried out on two separate days with each session lasting around three hours. In total, 15 participants took part in the experiment with $86.67 \%$ (13) male and $13.33 \%$ (2) female. $53.55 \%$ (8) of the participants fall between the age group of 25 to 34 years old, $40 \%$ (6) of them are between 18 to 24 years old and one of them is a mature student in the age group of 35 to 44 . Participants were randomly recruited during the day of the experiment. $53.33 \%$ (8) of the participants are students at Department of Computer Science and the remaining are students from other departments who are not familiar with this part of the building. There were four participants for group A (CompSci student with no PIPS assistance), three participants for group B (Non-CompSci student with no PIPS assistance), five participants for group C (CompSci student with PIPS assistance) and three participants for group D (Non-CompSci student with PIPS assistance).

In the survey, all respondents had experienced being lost in a building in the past. Preventatively, some of they would try to gather information about location either via the Internet or from information to get better direction (80.04\%:12). All participants were familiar with the use of mobile navigation apps and have used this to find their way around unfamiliar places. In the experiment, eight participants from group $\mathrm{C}$ and group $\mathrm{D}$ were given the PIPS as a navigational aid to find a given room. $87.5 \%$ (7) of the participants found the system useful and $75 \%$ (6) had expressed interest to use PIPS in the future. One of the participants did comment that PIPS was not necessary, but that was because the participant is familiar with the location and agreed that it would be helpful to people that are unfamiliar with the location. It was also observed that $75 \%$ (6) of the participants would not have known the location of the room without the aid of the PIPS. They knew where they were going and they did save time not having to backtrack or explore within the two floors. In terms of functionality, participants from both group $\mathrm{C}$ and group $\mathrm{D}$ found that selecting a room number and person name from the list was convenient. This prevents them from making mistakes from incorrect data input to PIPS. They also found the location marker placed around floors highly visible and the use of NFC tags to provide the current position useful.

The other seven participants from group A and group B (without PIPS assistance) who were given the same task to look for a location generally took longer to locate the room. $71.43 \%$ (5) of the participants had to resort to exploring the floor and searching the rooms one by one until the participant located the room, while another took a smarter approach by simply searching for rooms on one side of the corridor because the participant knew that the odd numbered rooms are located on the left of the corridor and even numbered rooms are on the right of the corridor. Another participant for asked a passer-by for direction.

It was interesting to observe that although they had been at the Department for some time and were familiar with the building, all of the participants from group A were still wayfinding their way around the floor. This is because most of the participants may not know everyone in the Department and may not have used all the common rooms before. Therefore they would have only developed a partial cognitive map of their area of interest. This explains why $75 \%$ (3) of participants from group C (CompSci students with PIPS assistance) found the PIPS useful and would use PIPS in the future. We believe 
this is clear evidence that PIPS remains useful for local residents as well as visitors to buildings.

When comparing participants from group B with group D, it is obvious that PIPS did provide the navigational aid to locate a room in a building. Without the assistance of PIPS, all of the participants in group D took longer to locate the room as they needed the time to explore the unfamiliar space. Even though they were based in the same building, it only gave them a slight advantage as they were able to borrow cognitive maps from other floors to wayfind efficiently. For new visitors, each floor and door has an almost identical appearance, and the lack of prominent landmarks within each floor will certainly require more time for one to find a specific location in the building. A further complication exists in that some rooms are hidden from the main corridor in the test environment.

\section{CONCLUSIONS AND FUTURE WORKS}

In this paper, we have surveyed the conventional methods of indoor navigation and looked into the use of IoT technologies as solution to provide directional assistance inside a building. The existing indoor positioning systems reviewed are viable solutions but have some limitations. Our proposed PIPS is a low cost and effective indoor navigation solution that can be scaled up easily. Our findings from the pilot experiment show that PIPS is useful to both people that are familiar with the location and those that are not. This could useful for application in locations such as airport where PIPS can provide travellers with useful direction to boarding gates, the estimated time required to reach the boarding gate and alerts on real-time flight schedules. This could potentially present huge impact on passenger experience and airliner to maintain on-time departure and arrival. Furthering our research in this area, we intend to expand PIPS to cover University buildings around the city; improve the UI; to include common places of interest; to allow node status to be updated dynamically via a web service; and to provide real-time positioning using on-board smartphone sensors.

\section{REFERENCES}

[1] "Activities conducted on a smartphone: teens", March 2011, Ofcom [Online], Avalilable: http://stakeholders.ofcom.org.uk/market-dataresearch/market-data/communications-market-reports/cmr11/uk/1.45 [Accessed 03 August 2015]

[2] "Activities conducted on a smartphone: adults", March 2011, Ofcom [Online], Available: http://stakeholders.ofcom.org.uk/market-dataresearch/market-data/communications-market-reports/cmr11/uk/1.44 [Accessed 03 August 2015]

[3] "The Internet of Things: How the Next Evolution of the Internet is Changing Everything", April 2011, D. Evans [Online], Available: https://www.cisco.com/web/about/ac79/docs/innov/IoT_IBSG_0411FIN AL.pdf

[4] F. Bacim, D. Bowman and M. Pinho, "Wayfinding techniques for multiScale virtual environments", , 2009. 3DUI 2009. IEEE Symposium on $3 D$ User Interfaces, pp.67,74, 14-15 March 2009, doi: 10.1109/3DUI.2009.48112074.

[5] P. Salmi, "Wayfinding Design: Hidden Barriers to Universal Access.", InformeDesign Where Research Informs Design, Vol. 5:9, August 2008, Available: http://www.informedesign.org/_news/aug_v05r-p.pdf

[6] R. Li and A. Klippel, "Using space syntax to understand knowledge acquisition and wayfinding in indoor environments," 9th IEEE International Conference on Cognitive Informatics (ICCI), pp.302,307, 7-9 July 2010, doi: 10.1109/COGINF.2010.5599724

[7] M.J. O’Neill, "Evaluation of A Conceptual Model Of Architectural Legibility”. Environment and Behavior, Vol. 23:3, pp. 259-284, May 1991.

[8] "IS07001:2007, Graphical Symbols - Public information symbols", 2013, ISO [Online], Available: http://www.iso.org/iso/catalogue detail?csnumber=41081
[9] "IS0 22727:2007:2007, Graphical Symbols -- Creation and design of public information symbols -- Requirements", 2013, ISO [Online], Available: http://www.iso.org/iso/catalogue_detail?csnumber $=41091$

[10] Y-S. Hung, K.-L.B. Chen, C-T. Yang; G.-F. Deng, Y-H Yeh and N-C $\mathrm{Wu}$, "Data mining for analysing kiosk usage behavior patterns," IEEE International Conference on Automation Science and Engineering (CASE) 2014, pp.1115,1120, 18-22 Aug. 2014, doi: 10.1109/CoASE.2014.6899465

[11] M.C. Maguire, “A Review of User-Interface Design Guidelines for Public Information Kiosk Systems", International Journal of HumanComputer Studies, vol.50:3, pp. 263-286, March 1999.

[12] G. Zhong and U. Hengartner, U., "A distributed k-anonymity protocol for location privacy", IEEE International Conference on Pervasive Computing and Communications (PerCom 2009), pp.1,10, 9-13 March 2009, doi: 10.1109/PERCOM.2009.4912774.

[13] M. Ashouri-Talouki, A. Baraani-Dastjerdi and A. A. Selcuk, "Preserving location privacy for a group of users", Turkish Journal of Electrical Engineering and Computer Sciences, vol 21:1, pp. 1857-1870, 2013.

[14] N. Krishnamurthy, "Using SMS to deliver location-based services", IEEE International Conference on Personal Wireless Communications, pp.177,181, 15-17 Dec. 2002, doi: 10.1109/ICPWC.2002.1177272

[15] A.H. Lashkari, B. Parhizkar and M.N.A. Ngan, "WIFI-Based Indoor Positioning System," Second International Conference on Computer and Network Technology (ICCNT2010), pp.76 -78, 23-25 April 2010, doi: 10.1109/ICCNT.2010.33

[16] J. Korhonen, T. Ojala, M. Klemola, and P. Vaanallen, "mTag Architecture for Discovering Location Specific Mobile Web Services Using RFID and Its Evaluation with Two Case Studies," . International Conference on Internet and Web Applications and Services/Advanced International Conference on Telecommunications(AICT-ICIW '06), pp.191,191, 19-25 Feb. 2006

[17] "Bluetooth Smart (Low Energy) Technology", n.d., Bluetooth SIG, [Online] Available: https://developer.bluetooth.org/TechnologyOverview/Pages/BLE.aspx [Accessed 03 August 2015]

[18] “How does it work?", 9 August 2012, Estimote, Available: http://estimote.com/ [Accessed 03 August 2015]

[19] “How it works?", n.d., StickNFind [Online] https://www.sticknfind.com/sticknfind.aspx?scrollTo=0 [Accessed 03 August 2015]

[20] "Tile", 2013, Tile [Online], Available: https://www.thetileapp.com/howit-works [Accessed 03 August 2015]

[21] Indiegogo. "Flic: The Wireless Smart Button", January 2015, Indiegogo [Online], Available: https://www.indiegogo.com/projects/flic-thewireless-smart-button [Accessed 03 August 2015]

[22] U. Bandara, M. Hasegawa, M. Inoue, M. Hiroyuki and T. Aoyama, "Design and implementation of a Bluetooth signal strength based location sensing system", IEEE Radio and Wireless Conference, pp.319,322, 19-22 Sept. 2004, doi: 10.1109/RAWCON.2004.1389140

[23] R. Musaloiu-E. and A. Terzis, "Minimising the effect of WiFi interference in 802.15.4 wireless sensor networks", International Journal of Sensor Networks, vol. 3:1, pp 43-54, 2008.

[24] Y-H. Chang, C-H. Chu and M-S. Chen, "A General Scheme for Extracting QR Code from a Non-uniform Background in Camera Phones and Applications," Ninth IEEE International Symposium on Multimedia (ISM 2007), pp.123,130, 10-12 Dec. 2007, doi: 10.1109/ISM.2007.4412365

[25] Want, R., "Near field communication" , IEEE Pervasive Computing, vol.10, no. 3, pp.4,7, July-September 2011, doi: 10.1109/MPRV.2011.55

[26] Want, R., "An introduction to RFID technology," Pervasive Computing, IEEE , vol.5, no.1, pp.25,33, Jan.-March 2006, doi: 10.1109/MPRV.2006.2

[27] "One in three mobile phones to come with NFC by 2017", $5^{\text {th }}$ June 2013, S. Clark [Online], Available: http://www.nfcworld.com/2013/06/05/324448/one-in-three-mobilephones-to-come-with-nfc-by-2017/ [Accessed 03 August 2015]

[28] F. Riaz and K.M. Ali, "Applications of Graph Theory in Computer Science," Computational Intelligence, Third International Conference on Communication Systems and Networks (CICSyN) 2011, pp.142,145, 26-28 July 2011, doi: 10.1109/CICSyN.2011.40

[29] N, Carey, "Establishing Pedestrian Walkng Speeds", Portland State University, $31^{\text {st }}$ May 2005, Available: http://www.step.groups.pdx.edu/docs/data_collection/ITE_Data_Collecti on_PSU_2005.pdf [Accessed 03 August 2015] 